$>$ Le cervelet, partie du cerveau qui coordonne la fonction motrice, a un rôle clé dans l'apprentissage moteur, voire dans certains processus cognitifs. II est aujourd'hui couramment admis que la modification à long terme de l'efficacité de la transmission synaptique est la base cellulaire de la mémoire et de l'apprentissage. La cellule de Purkinje, élément central de l'organisation cytoarchitectonique du cervelet et de la fonction cérébelleuse, est le siège d'une plasticité neuronale particulière appelée « dépression à long terme». En quoi consiste cette «dépression »? Quels en sont les mécanismes cellulaires et moléculaires? Quels éléments permettent de considérer que cette plasticité est le mécanisme de base de l'apprentissage moteur? Telles sont les questions abordées dans cet article. <

\section{La dépression synaptique à long terme: un mécanisme pour la mémoire et 'apprentissage au niveau du cervelet}

Réjan Vigot

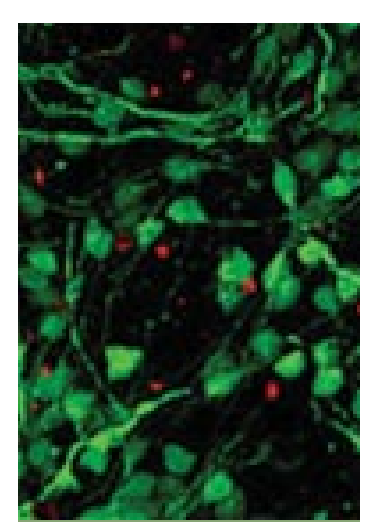

fonctionnement du cervelet $[3,4]$. Cette région du cerveau assure la coordination motrice et doit intégrer les différents messages sensoriels, de manière à régler finement les mouvements $d u$ corps, de la tête et des extrémités. De plus, la structure du cervelet est maintenant bien connue et son implication dans des fonctions supérieures, cognitives, paraît assurée. Il constitue donc un modèle de choix dans l'étude des mécanismes de la mémoire et de l'apprentissage $(\rightarrow)$.

\section{Le cervelet est connecté en parallèle sur les voies motrices}

D'un point de vue anatomique, le cervelet est une structure médiane impaire qui se trouve sur la face dorsale du tronc cérébral. II est rattaché à ce dernier par trois pédoncules individualisés par lesquels transitent les afférences et les efférences cérébelleuses. Les afférences majeures sont les fibres moussues et les fibres grimpantes. Les premières, ayant pour origine les neurones de nombreux relais précérebelleux, convoient des informations en provenance des différentes parties du corps. Les fibres grimpantes ont une 
origine unique: les neurones de l'olive inférieure, située dans la partie ventro-médiane du bulbe. Les efférences cérébelleuses motrices gagnent les noyaux vestibulaires, rouges et thalamiques, ainsi que les centres des voies réticulo-spinales. D'autres efférences, faisant partie des circuits de rétrocontrôle, sont dirigées vers l'olive inférieure. Les cellules de Purkinje constituent l'élément central du cortex cérébelleux et de la fonction cérébelleuse (Figure 1)

$(\rightarrow) \mathrm{m} / \mathrm{s}$ $2000, n^{\circ} 4$, p. 500 $(\rightarrow)$. Leur axone forme l'unique efférence corticale et c'est vers ces neurones que convergent les diverses afférences cérébelleuses. Les cellules de Purkinje sont ainsi au contact de deux afférences excitatrices (glutamatergiques) majeures: les fibres parallèles (partie distale des axones des cellules granulaires) et les fibres grimpantes. Les premières relaient l'information transmise par les fibres moussues; les secondes sont en contact direct, et semble-t-il exclusif, avec les cellules de Purkinje de l'hémi-cervelet controlatéral, selon une topographie précise [5]. Chez l'adulte, chaque cellule de Purkinje est au contact d'un grand nombre de fibres parallèles et d'une seule fibre grimpante. Le cortex cérébelleux contient aussi d'autres neurones (interneurones inhibiteurs) que sont les cellules étoilées, les cellules en corbeille ainsi que les cellules de Golgi. Après intégration neuronale, les axones des cellules de Purkinje vont inhiber l'activité des neurones des noyaux cérébelleux, dont les axones forment les efférences cérébelleuses.

\section{Mécanismes cellulaires et moléculaires de la dépression à long terme}

La dépression à long terme est principalement un phénomène hétérosynaptique, résultant de modifications intervenant dans la partie post-synaptique et ne semblant pas mettre en jeu de modifications de la partie pré-synaptique $[4,6]$. Les voies intra- et intercellulaires, ainsi que les différentes molécules impliquées dans l'induction de la dépression à long terme, sont schématisées sur la Figure 2. L'enregistrement électrophysiologique des cellules de Purkinje permet de suivre en continu l'évolution de la dépression à long terme dans le temps pour des durées de une à deux heures in vitro, voire de cinq heures chez l'animal décérébré [7]. L'idée actuelle est que l'activation des différentes voies intracellulaires conduit à la phosphorylation des récepteurs AMPA (récepteurs ionotropiques du glutamate) des cellules de Purkinje, conférant une moindre sensibilité de ces neurones à l'activation par les fibres parallèles.

La phosphorylation des récepteurs AMPA est-elle suffisante pour expliquer le maintien à plus long terme de la dépression synaptique? Des modifications plus profondes ont-elles lieu? Ces dernières pourraient consister, entre autres, en une modification de l'expression génique après

\section{La dépression à long terme est produite par les erreurs faites au cours de l'apprentissage}

Le schéma des circuits cérébelleux (Figure I) se répète à l'identique dans l'ensemble du cervelet. II décrit aussi l'unité fonctionnelle de base de la fonction cérébelleuse, le micro-complexe, dans lequel chaque micro-zone corticale est en relation avec des neurones distincts des noyaux cérébelleux ou vestibulaires, ainsi que de l'olive inférieure. Le fonctionnement du micro-complexe, étendu au cervelet, est modélisé de la manière suivante: l'information transitant par les fibres moussues/parallèles est une représentation de la tâche à accomplir; si, au cours de l'apprentissage, celle-ci n'est pas effectuée correctement, un «signal d'erreur », émis en temps réel, d'origine sensorielle ou centrale, parvient aux cellules de Purkinje par l'afférence grimpante, afin de diminuer le poids des synapses fibre parallèle-cellule de Purkinje actives de manière inappropriée. La dépression à long terme cérébelleuse consiste ainsi en une diminution de l'efficacité de la transmission des synapses fibre parallèle-cellule de Purkinje à la suite de l'activation conjointe du neurone par ses afférences parallèle et grimpante. Les signaux d'erreur causés par une action inadéquate conduisent à la dépression à long terme, qui modifie alors le circuit neuronal de manière à diminuer les erreurs.

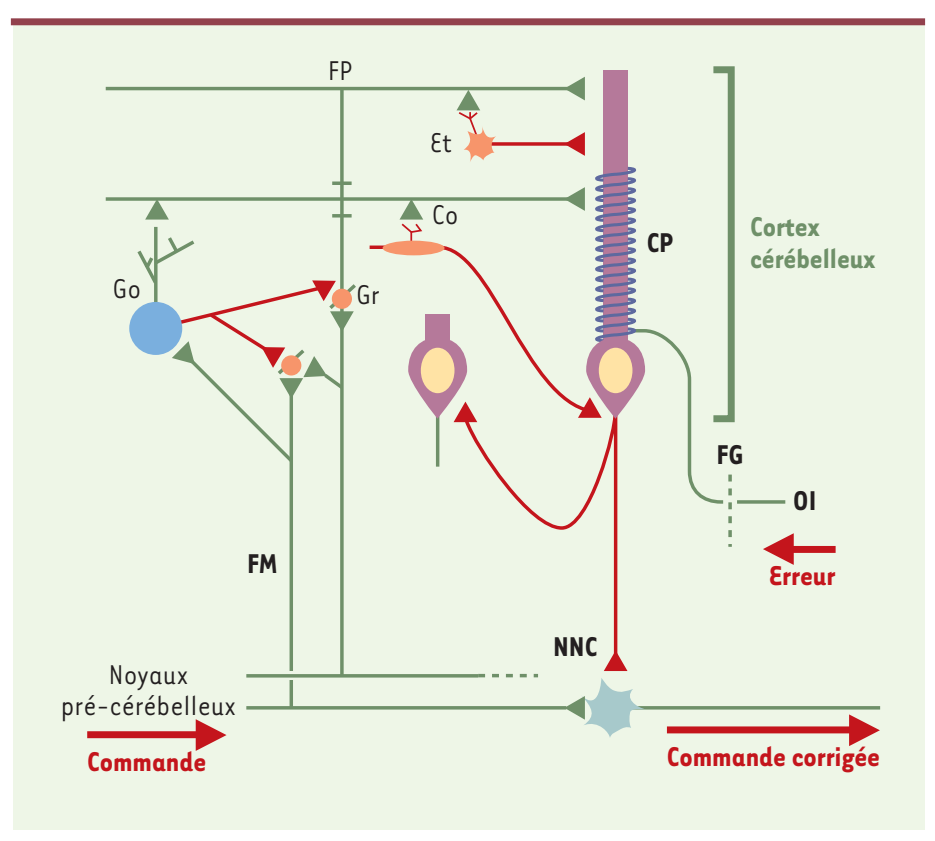

Figure 1. Représentation schématique des circuits cérébelleux. Les neurones excitateurs et leurs terminaisons sont représentés en vert, les inhibiteurs en rouge. Co: cellules en corbeille; $C P$ : cellules de Purkinje; $\varepsilon t$ : cellules étoilées; FG: fibres grimpantes; FM: fibres moussues; FP: fibres parallèles; Go: cellules de Golgi; Gr: cellules granulaires; NNC: neurones des noyaux cérébelleux; 01 : olive inférieure. 
l'induction de la dépression à long terme. II a effectivement été montré que des conditions expérimentales compatibles avec l'induction de la dépression à long terme peuvent provoquer une augmentation de l'expression de certains gènes, tels que celui codant pour le BDNF (brain- derived neurotrophic factor) ou le gène précoce jun- $B$. La synthèse de nouvelles molécules pourrait être une autre manière de stabiliser la dépression à long terme après son induction. Cela a été mis en évidence par D.J. Linden [9], qui montra que l'arrêt de la synthèse protéique conduit uniquement à une « dépression à long terme » de courte durée, et par

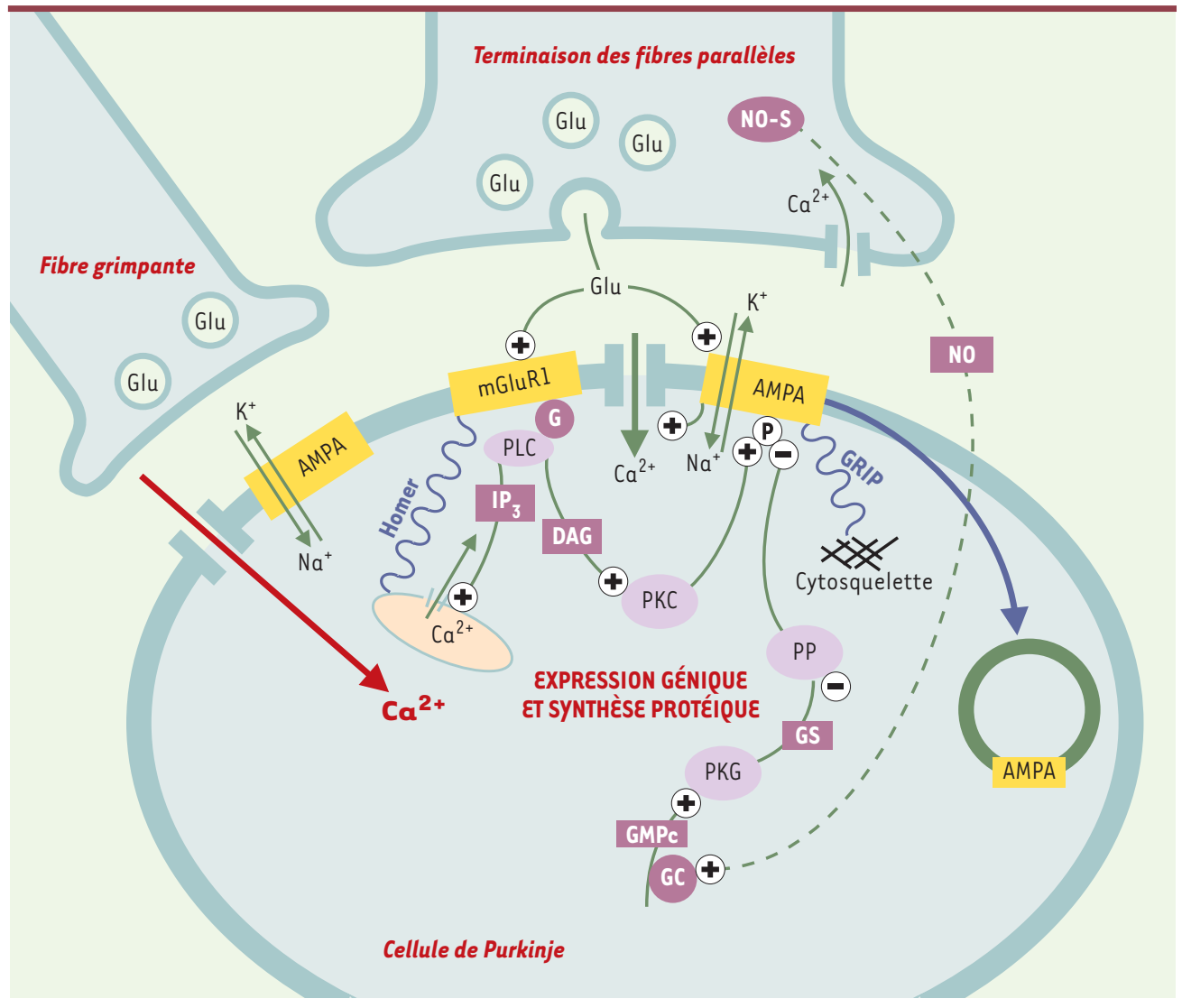

Figure 2. Représentation schématique des mécanismes cellulaires et moléculaires de la dépression à long terme. Le glutamate libéré par les terminaisons des fibres grimpantes (principalement) et parallèles active les récepteurs AMPA, provoquant ainsi la dépolarisation du neurone et l'ouverture de canaux calciques dépendant du potentiel. L'augmentation du $\mathrm{Ca}^{2+}$ libre intracellulaire, liée à l'ouverture de ces canaux et, éventuellement, à la libération de $\mathrm{Ca}^{2+}$ à partir des réserves intracellulaires [8], est un facteur essentiel pour l'induction de la dépression à long terme. Le glutamate des fibres parallèles active aussi les récepteurs métabotropiques du sous-type l (mGluRl), provoquant l'activation de la phospholipase $C(P L C)$ et la formation de diacylglycérol et d'inositol-triphosphate. Ce dernier induit, après l'activation de ses récepteurs, la libération de $\mathrm{Ca}^{2+}$ à partir des stocks intracellulaires. Deux protéines kinases, la protéine kinase C (PKC) et la protéine kinase G (PKG), ont un rôle central dans le contrôle de l'état de phosphorylation des récepteurs AMPA: la PKC phosphoryle directement les récepteurs après son activation par le diacylglycérol tandis que la PKG est parallèlement impliquée dans l'inhibition de la déphosphorylation de ces mêmes récepteurs. La voie intracellulaire mettant en jeu cette seconde kinase est activée par un neuromédiateur diffusible, le NO (monoxyde d'azote), qui est synthétisé et libéré par les terminaisons des fibres parallèles. Sa diffusion au sein de la cellule de Purkinje provoque l'activation de la guanylate cyclase et la formation de GMP cyclique. Ce dernier est l'activateur de la PKG qui inactive les phosphatases par l'intermédiaire d'un substrat spécifique, le $G$-substrate. AMPA: récepteurs ionotropiques glutamatergiques de type AMPA; DAG: diacylglycérol; G: protéine G; GC: guanylate cyclase; Glu: glutamate; GMPc: GMP cyclique; GRIP: glutamate receptor interacting protein; GS: G-substrate; Homer: protéine codée par le gène homer; IP3: inositol-triphosphate; $\mathrm{mGluRl}$ : récepteurs métabotropiques glutamatergiques de type 1; N0: monoxyde d'azote, NO-S: NO-synthase; P: phosphate (phosphorylation); PKC: protéine kinase C; PKG: protéine kinase G; PLC: phospholipase C; PP: protéines phosphatases. Les signes + indiquent une activation, les - une inactivation. une étude plus récente montrant que l'intégrité de la transcription et celle de la traduction sont en fait également nécessaires à l'induction de la dépression à long terme [10]. Ces résultats indiquent que la synthèse protéique dans les cellules de Purkinje est un élément clé de l'induction et du maintien à plus long terme de la dépression à long terme. La nature et le lieu de synthèse (soma/dendrite) de la (ou des) protéine(s) restent toutefois à être définis.

La quantité et la distribution des protéines membranaires sont modulées par des processus de fusion membranaire (enrichissement) et d'endocytose (internalisation). La localisation spécifique des récepteurs dans les zones actives des membranes post-synaptiques est assurée par des molécules d'ancrage qui ont aussi pour rôle de mettre en étroite relation les récepteurs et les voies de signalisation. II s'agit en particulier de la protéine GRIP (glutamate receptor interacting protein), étroitement associée aux sousunités GluR2 des récepteurs AMPA dans les dendrites des cellules de Purkinje, et de la protéine Homer, qui couple les récepteurs membranaires mGluRl aux récepteurs de 
l'inositol-triphosphate du réticulum. Inversement, l'endocytose est liée à la formation de vésicules couvertes de $(\rightarrow) \mathrm{m} / \mathrm{s}$ $2000, n^{\circ} 3$, p. 440 clathrine $[11](\rightarrow)$. La dépression à long terme est aussi le résultat d'une internalisation des récepteurs AMPA postsynaptiques. En effet, l'inhibition de la formation des complexes d'endocytose empêche l'induction de la dépression à long terme [12]; l'induction de la dépression à long terme et l'activation de l'endocytose s'excluent mutuellement [12]. De plus, l'induction de la dépression à long terme s'accompagne d'une modification de la distribution et de l'internalisation des sousunités GluR2 [13]. Sous l'effet de la phosphorylation de la protéine GRIP par la PKC (protéine kinase C), une partie significative des récepteurs AMPA quitteraient finalement la zone active de la membrane post-synaptique pour être internalisés [14].

Notons toutefois que le modèle et les mécanismes décrits ci-dessus sont une représentation simplifiée des modèles et des mécanismes proposés actuellement. La dépression à long terme hétérosynaptique est un exemple, certes majeur, de plasticité synaptique parmi d'autres se produisant au sein des circuits cérébelleux: la dépression à long terme homosynaptique (causée par une importante activation des cellules de Purkinje par les seules fibres parallèles), la potentialisation à long terme pré-synaptique des synapses fibres parallèles-cellules de Purkinje, et la potentialisation à long terme des synapses fibres moussues-cellules granulaires, voire la dépression à long terme des synapses fibres grimpantes-cellules de Purkinje.

\section{Dépression à long terme et apprentissage}

Les manipulations pharmacologiques et génétiques utilisées pour étudier les mécanismes cellulaires et moléculaires de la dépression à long terme ont aussi permis de lier plus étroitement dépression à long terme et apprentissage moteur, comme l'a récemment souligné Ito [4]. Nous présenterons ci-après quelques résultats montrant que l'absence de dépression à long terme est associée à un dysfonctionnement, voire une absence de la faculté d'apprentissage.

Le réflexe vestibulo-occulaire (ou VOR) est étroitement contrôlé par le cervelet. II provoque le mouvement des yeux destiné à compenser le mouvement de la tête. Des prismes sont utilisés pour modifier la perception de l'amplitude et/ou de la direction du mouvement de la cible. L'animal doit adapter son VOR conformément à la nouvelle configuration. Alors que le monoxyde d'azote (NO) est nécessaire à l'induction de la dépression à long terme, sa suppression ou l'arrêt de sa synthèse par le cervelet empêche l'adaptation du VOR $(\rightarrow)$. De même, l'inhibition continue de la PKC, chez la souris transgénique, dont les cellules de Purkinje expriment un inhibiteur de la PKC [15], conduit à une absence de dépression à long terme et à une absence d'adaptation du VOR. Parallèlement, la manipulation du NO cérébelleux chez le singe provoque une altération de l'adaptation de la poursuite visuelle d'une cible dont la vitesse de déplacement varie. Le conditionnement du clignement de la paupière (destiné à protéger l'œil) fait lui aussi partie des comportements liés au fonctionnement du cervelet. II s'agit pour l'animal de fermer sa paupière lorsqu'un son, préalablement associé à un bref jet d'air dirigé vers l'œil, lui est présenté. L'altération des mécanismes de la dépression à long terme conduit là encore à une altération dans l'apprentissage de ce conditionnement. De plus, dans ce type d'expérience, et dans d'autres, l'activité des fibres grimpantes est accrue en cas d'erreur et retourne à un niveau basal au cours de l'acquisition. Des altérations de la locomotion et de la coordination motrice apparaissent également lorsque les mécanismes de la dépression à long terme ou la structure du micro-complexe sont perturbés. Par exemple, lors de la marche, l'animal peut adapter le mouvement d'une extrémité indépendamment de celui des autres. Une telle adaptation est rendue impossible chez le chat dont la synthèse de NO cérébelleux est inhibée [16], et chez la souris dépourvue de récepteurs mGluRl [17]. Mais, dans le second cas, la dépression à long terme, tout comme la coordination motrice et l'apprentissage moteur, peuvent être restaurés par le seul rétablissement de l'expression de mGluRl dans les cellules de Purkinje [17].

\section{Conclusions}

La modification à long terme de l'efficacité de la transmission synaptique est aujourd'hui considérée comme l'un des mécanismes cellulaires de la mémoire et de l'apprentissage. Dans le cervelet, il s'agit plus particulièrement d'une dépression, conséquence des échecs ayant lieu au cours de l'apprentissage. Si le modèle présenté apparaît valide, il reste cependant très incomplet lorsqu'il s'agit de définir si la dépression à long terme se maintient au cours du temps et est convertie en une forme de mémoire plus permanente. L'implication du cervelet dans certaines tâches cognitives est suggérée par des observations cliniques ainsi que par les résultats d'études non invasives. II est séduisant d'extrapoler le modèle de l'apprentissage moteur à l'apprentissage en général. Cependant, des progrès importants restent à faire et des études plus nombreuses sur l'animal vigile et le sujet humain sont nécessaires pour mieux comprendre les mécanismes de l'apprentissage et de la mémoire. $\diamond$ 


\section{SUMMARY}

Cerebellar long-term depression: a mechanism for learning and memory

It is commonly thought that a persistent change in the efficacy of the synaptic transmission is the basic mechanism underlying learning and memory. The cerebellum, key structure of the motor function, exhibits a synaptic plasticity named cerebellar long-term depression or LTD. This phenomenon appears in the Purkinje cell when the two main excitatory inputs (one consists of the parallel fibers which relay information on the task to accomplish and the other one includes the climbing fiber which conveys error signals) are activated in combination, resulting in a persistent decrease of the efficacy of the parallel fiberPurkinje cell synapse. Studies made in the last 20 years show that activation of ionotropic and metabotropic glutamate receptors triggers complex signal transduction processes, leading to the phosphorylation and the internalization of AMPA receptors, a subtype of glutamatergic receptors. The aim of this paper is firstly to present mechanisms involved in LTD induction and maintenance. The second part introduces briefly experimental data that show that LTD is indeed strongly associated with motor learning. Recent studies on the involvement of the cerebellum in cognitive tasks also suggest that LTD may play some role other than that in the sole motor learning. $\diamond$

\section{RÉFÉRENCES}

1. Bliss TVP, Gardner-Medwin AR. Long-lasting potentiation of synaptic transmission in the dentate area of the unanesthetized rabbit following stimulation of the perforant path. J Physiol 1973; 232: 357-74.

2. Ito $M$, Sakurai $M$, Tongroach P. Climbing fibre induced depression of both mossy fibre responsiveness and glutamate receptors in cerebellar Purkinje cells. J Physiol 1982; 324: 113-34.

3. Ito M. The cerebellum and neural control. New York: Raven Press, 1984.

4. Ito M. Cerebellar long-term depression: characterization, signal transduction, and functional roles. Physiol Rev 2001; 81: 1143-95.

5. Voogd J. Cerebellum. In: Paxinos G, ed. The rat nervous system. $2^{\text {nd }} \mathrm{ed}$. San Diego: Academic Press, 1995 : 309-44.

6. Levenes C, Daniel H, Crépel F. Long-term depression of synaptic transmission in the cerebellum: cellular and molecular mechanisms revisited. Prog Neurobiol 1998; 55: 79-91.

7. Vigot R, Batini C, Kado RT, Yamamori T. Synaptic longterm depression (LTD) in vivo recorded on the rat cerebellar cortex. Arch Ital Biol 2002; 140: 1-12.

8. Daniel H, Levenes C, Fagni L, Conquet $\mathrm{F}$, Bockaert J, Crépel F. Inositol-1,4,5trisphosphate-mediated rescue of cerebellar longterm depression in subtype 1 metabotropic glutamate receptor mutant mouse. Neuroscience 1999; 92: 1-6.

9. Linden DJ. A protein synthesis-dependent late phase of cerebellar longterm depression. Neuron 1996; 17: 483-90.
10. Karachot L, Shirai $Y$, Vigot $\mathrm{R}$, Yamamori T, Ito $\mathrm{M}$. Induction of long-term depression in the cerebellar Purkinje cells requires a rapidly turned over protein. J Neurophysiol 2001; 86: 280-9.

11. Jarousse N, Kelly RB. Endocytotic mechanisms in synapses. Curr Opin Cell Biol 2001; 13: 461-9.

12. Wang $Y \mathrm{~T}$, Linden DJ. Expression of cerebellar long-term depression requires postsynaptic clathrin-mediated endocytosis. Neuron 2000; 25: 635-47.

13. Matsuda $S$, Launey T, Mikawa S, Hirai H. Disruption of AMPA receptor GluR2 clusters following long-term depression induction in cerebellar Purkinje neurons. EMBO / 2000; 19 : 2765-74.

14. Hirai H. Modification of AMPA receptor clustering regulates cerebellar synaptic plasticity. Neurosci Res 2001; 39: 261-7.

15. De Zeeuw Cl, Hansel C, Bian F, et al. Expression of a protein kinase $C$ inhibitor in Purkinje cells blocks cerebellar LTD and adaptation of the vestibulo-ocular reflex. Neuron 1998; 20 : 495-508.

16. Yanagihara D, Kondo I. Nitric oxide plays a key role in adaptative control of locomotion in cat. Proc Natl Acad Sci USA 1996; 93: 13292-7.

17. Ichise T, Kano M, Hashimoto K, et al. mGluRl in cerebellar Purkinje cells essential for long-term depression, synapse elimination and motor coordination. Science 2000; 288: 1832-5.

\section{TIRÉS À PART}

R. Vigot 